\title{
A Review of Autonomous Buildings of the Future: Wisdom on Sustainable Design in Architecture and Engineering
}

\author{
Yonas Ahmed Mohammed* \\ University of Adelaide, South Australia
}

*Corresponding author: Yonas Ahmed Mohammed, University of Adelaide, South Australia.

Received Date: April 02, 2019

Published Date: April 17, 2019

\section{Opinion}

Imagine a building that operates independent of our urban energy grids. A building that automatically adapts its behavior to the needs of each occupant and user, intelligently balancing to the preferences of the owner. A building that configures itself to provide a separate energy source for its consumption, health and safety in the most appropriate way considering all the relevant risks and conditions. A building not only functional and efficient but offers alternative lifestyle that improves productivity. An autonomous building is a type of building that automatically reaches out to our nearest available resources and engages within its provided financial budget and with minimal artificial human intervention and customization. The road to autonomy is a slow journey that starts by breaking down building systems into a network of smaller components. These systems would collect and analyze a wide variety of data, enabling physical buildings to be managed, easily understood and reconfigured as needed. The autonomous building construction system maximizes the ratings in each of the building classification systems. Due to the fact that the main impact of buildings on the external environment is their energy demand during their use, hence, autonomous buildings significantly reduce energy demand during and after construction.

British architects Brenda and Robert Vale said that "It is quite possible in all parts of Australia to construct a 'house with no bills', which would be comfortable without heating and cooling, which would make its own electricity, collect its own water and deal with its own waste...These houses can be built now, using off-the-shelf techniques. It is possible to build a "house with no bills" for the same price as a conventional house, but it would be by one-fourth smaller in size". As an architect or engineer would be concerned with the shortcomings of transportation networks, and dependence on distant resources, as the design of autonomous buildings tend to include more autonomous features. Our attempts to make our buildings more autonomous has been faced with an apprehension for sources of heat, power, water and food. A nearly parallel path toward autonomy has been to start with a concern for environmental impacts. Autonomous buildings definitely reduce environmental impacts by using on-site resources such as sunlight and rain that would otherwise be left unused. Autonomy radically reduces the costs and impacts of networks that serve the building.

An autonomous house in concept must be custom-built to suit the climate, site and location. Passive solar techniques, alternative toilet and sewage systems, thermal massing designs, battery systems, efficient windowing, and the array of other design tactics require some degree of non-standard construction, added expense, ongoing experimentation and maintenance, and also have an effect on the psychology of the space, in a way new forms of architectural and engineering experimentation. An autonomous structure is not always environmentally friendly. The goal of independence from support systems is associated with, but not identical to, other goals of environmentally responsible green building. However, autonomous buildings also usually include some degree of sustainability through the use of renewable energy and other renewable resources, producing no more greenhouse gases than they consume, and other measures. Experts say no building will be truly autonomous. Architects and engineers will have to change their thinking a bit to understand how to tune and manage these components and systems. These tools are still complicated and need to be used by an expert to get the most benefit. It would take some 
time for a regular individual to live in an autonomous building. In the exploration of architectural Metabolism, elements that goes into the functioning of buildings, modern developments have brought the autonomy goals within reach, increasing the ability of modern data science to model and predict usage patterns, how consumer attitudes have grown to welcome bold deployments of automated technology and the development of semi-autonomous technologies that can be applied to buildings.

The traditional argument in favor of autonomous buildings attempts to show that the distribution networks in our city grids have larger inefficiencies i.e. a cost of continuing waste and capital expenses. Autonomous buildings simply provide the service with the building, hence, the usual counter-argument is that those inefficiencies and capital expenses are easily borne in urban concentrations where we heavily rely on urban grids. So, why isn't there substantial progress toward autonomous buildings, particularly when so much progress is being made regarding other autonomous technology, such as autonomous vehicles? After all, the challenges of operationalizing autonomous cars appear much greater than operationalizing stationary buildings. Architects and engineers will soon have to surpass their current set of skills to understand the future users' behavioral patterns and cycles and adapt their building conception accordingly to the idea of autonomy. By taking responsibility for more than just the structure, aesthetics and built properties of a project and considering the details of how a building functions day-to-day throughout its life cycle, we need to envision a future in which engineering would be responsible for assessing, responding to and ultimately determining the functional relationship between humanity and the buildings we interact with in every facet of our daily lives.

\section{Acknowledgment}

None.

\section{Conflict of Interest}

No conflict of interest. 